\title{
Study on Performance of Adaptive Maximum Torque Per Amp Control in Induction Motor Drives at Light Load Operation
}

\author{
Chun-Ki Kwon*, Yong-Hae Kong* and Dong-Sik Kim ${ }^{\dagger}$
}

\begin{abstract}
Efficient operation of induction motor at light loads has been getting wide attention recently because the operating of induction motor at light loads occupies big portion of its operating regions in many applications such as environment friendly vehicle. As one of approaches to improve efficiency, Adaptive Maximum Torque Per Amp (Adaptive MTPA) control for induction motor drives has been proposed to achieve a desired torque with the minimum possible stator current. However, the Adaptive MTPA control was validated only at heavy load where, in general, control scheme tends to perform better than at light loads since the error in measurement of sensors is lower and signal to noise is better. Thus, although the performance of a control scheme is good at rated operating point, its performance at light load is somewhat in doubt in practice. This has led to considerable interest in efficiency of Adaptive MTPA control at light loads. This work experimentally demonstrates performance of Adaptive MTPA control at light loads regardless of rotor resistance variation, thus showing its good performance over all operating conditions.
\end{abstract}

Keywords: Induction motor model, Maximum torque amp (MTPA) condition, Adaptive maximum torque per amp (Adaptive MTPA) control, Light load

\section{Introduction}

In the past years, commercial electric source from wall outlet has been in main source to drive induction motors at the rated load. As semiconductor technology has been developed, inverter has been widely used to drive induction motors. Because of inverter capable of drive induction motor at all operating points, the operation at light or mid ranges of loads has been increased along with demand of eco-friendly vehicles such as electric vehicle $[1,2]$. Thus, demand of highly efficient operation in induction motor drives at light or mid loads has been increased [1,2].

Highly efficient operation of induction motor has been studied in the past years [3-9]. Among many efforts to obtain efficient operation, an Adaptive Maximum Torque Per Amp (Adaptive MTPA) control of induction motor drives was validated to drive induction motor in highly efficient way $[7,8]$. That is because it employed an alternate $q d$ induction machine model (AQDM) rather than the classical $q d$ model (CQDM), such as in [9-13].

However, the Adaptive MTPA control strategy set forth in $[7,8]$ has demonstrated the ability to achieve the commanded torque with good accuracy regardless of temperature variation at the rated load where induction motor experiences rarely compared to light or mid loads. No study has been conducted on the optimality of the Adaptive MTPA control in the operating points of light or

$\dagger$ Corresponding Author: Dept. of Electrical Engineering, Soonchunhyang University, Korea. (dongsik@sch.ac.kr)

* Dept. of Medical Information Technology Engineering, Soonchunhyang University, Korea. (\{chunkikwon, yhkong\}@sch.ac.kr)

Received: February 9, 2016; Accepted: August 18, 2016

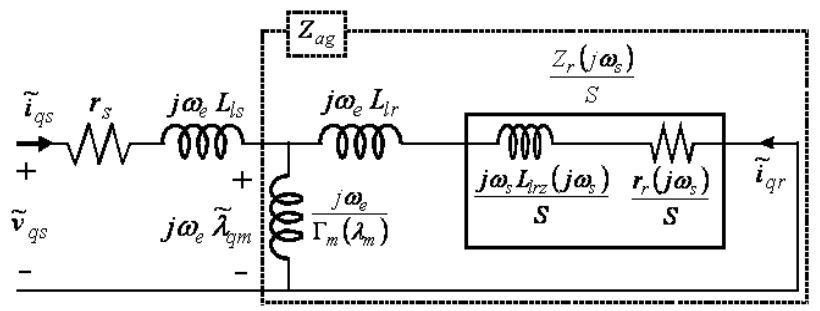

Fig. 1. Steady-state equivalent circuit of AQDM model

mid loads.

Thus, this work demonstrates its optimality in performance at light load in a way that torque command was generated with minimum stator current as rotor resistance varies.

\section{Alternate QD Induction Machine (AQDM)}

Alternate QD Model (AQDM) simultaneously included magnetic nonlinearties and the distributed nature of rotor circuits which CQDM in [9-13] has deficiency in representing over all possible operating conditions due to its constant parameters. Stator and rotor leakage inductance, and the absolute inverse magnetizing inductance are expressed as function of magnitude of magnetizing flux linkage, $\lambda_{m}$, which is equal to $\sqrt{2}\left|\tilde{\lambda}_{q m}\right|$, and are denoted as $L_{l s}\left(\lambda_{m}\right), L_{l r}\left(\lambda_{m}\right)$, and $\Gamma_{m}\left(\lambda_{m}\right)$, respectively. To consider distributed system effects in the rotor circuits, the rotor impedance, $Z_{r}\left(j \omega_{s}\right)$, is expressed in Laplace form and separated into a real and imaginary part, which are 
Table 1. Resultant parameters

\begin{tabular}{c|c|c|c|c|c}
\hline \multicolumn{2}{c|}{$L_{l s}(\cdot)$} & \multicolumn{2}{c|}{$\Gamma_{m}(\cdot)$} & \multicolumn{2}{c}{$Y_{r}(\cdot)$} \\
\hline$l_{s 1}$ & $9.06 \mathrm{e}-4$ & $m_{1}$ & $6.79 \mathrm{e} 0$ & $y_{a 1}$ & $5.65 \mathrm{e} 0$ \\
\hline \multicolumn{2}{|c|}{$l_{s 1}(\cdot)$} & $m_{2}$ & $6.62 \mathrm{e}-1$ & $y_{b 1}$ & $3.21 \mathrm{e}-2$ \\
\hline$l_{r 1}$ & $1.40 \mathrm{e}-4$ & $m_{3}$ & $5.03 \mathrm{e} 0$ & $y_{a 2}$ & $4.40 \mathrm{e}-2$ \\
\hline$l_{r 2}$ & $4.15 \mathrm{e}-3$ & $m_{4}$ & $1.85 \mathrm{e} 0$ & $y_{b 2}$ & $4.78 \mathrm{e}-4$ \\
\hline$l_{r 3}$ & $7.35 \mathrm{e}-1$ & $m_{5}$ & $8.68 \mathrm{e}-1$ & $y_{b 3}$ & $3.17 \mathrm{e}-3$ \\
\hline$l_{r 4}$ & $2.59 \mathrm{e} 0$ & $m_{6}$ & $1.29 \mathrm{e}-1$ & $y_{b 3}$ & $8.76 \mathrm{e}-8$ \\
\hline
\end{tabular}

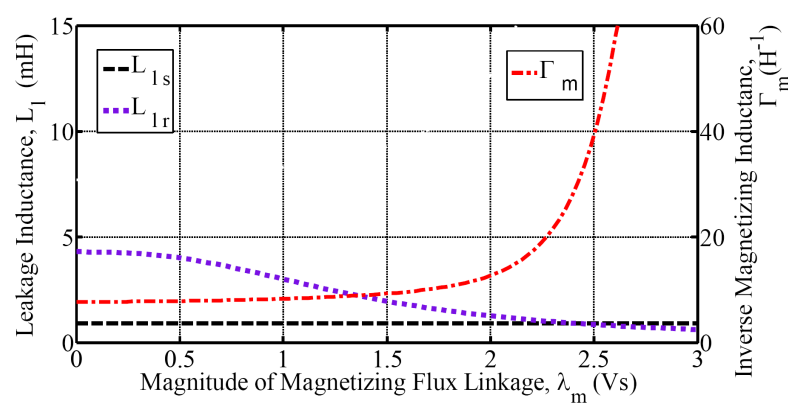

(a) Stator/Rotor leakage inductance and inverse magnetizing inductance

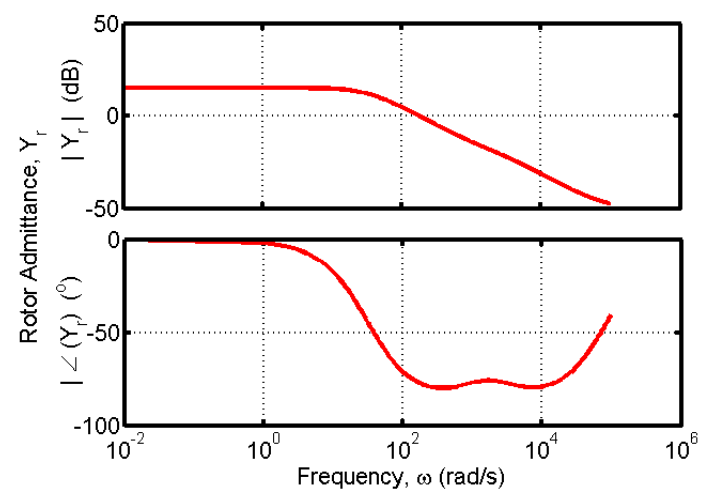

(b) Rotor admittance

Fig. 2. AQDM parameters for the test induction motor

denoted $r_{r}\left(j \omega_{s}\right)$ and $j \omega_{s} L_{l r z}\left(j \omega_{s}\right)$, respectively.

The steady-state equivalent circuit representing the AQDM in [14] is shown in Fig. 1. Additional details on the AQDM model and its nomenclature are found in [14-16]. In this work, the functional forms for AQDM parameters are specified as follows:

$$
\begin{gathered}
L_{l s}=l_{s 1} \\
L_{l r}\left(\lambda_{m}\right)=l_{r 1}+\frac{l_{r 2}}{1+\left(l_{r 3} \lambda_{m}\right)^{l_{r 4}}} \\
\Gamma_{m}\left(\lambda_{m}\right)=m_{1}-m_{2} \lambda_{m}+e^{m_{3}\left(\lambda_{m}-m_{4}\right)}+e^{m_{5}\left(\lambda_{m}-m_{6}\right)} \\
Y_{r}\left(s=j \omega_{s}\right)=\frac{1}{Z_{r}\left(s=j \omega_{s}\right)}=\frac{y_{a 1}}{y_{b 1} s+1}+\frac{y_{a 2}}{y_{b 2} s+1}+\frac{y_{a 3}}{y_{b 3} s+1}
\end{gathered}
$$

The parameters in (1) - (4) of AQDM were characterized by applying fitting process to the functional forms of (1) -
(4) on the laboratory experimental data taken for a 4-pole, $460 \mathrm{~V}, 50 \mathrm{Hp}, 60 \mathrm{~Hz}$, delta-connected squirrel cage induction motor. The resultant parameters in (1)-(4) of AQDM for the test induction motor are listed in Table 1 and are illustrated in Fig. 2. Detailed procedures of the parameter identification for AQDM is set forth in [14-16] but is omitted due to limited space.

\section{Adaptive Maximum Torque per Amp Control Strategy}

For convenience purpose, the procedure to design Adaptive MTPA control is rewritten in this section. More details can be referred to $[7,8]$.

\subsection{Objective and structure}

As shown in Fig. 3, Adaptive MTPA control strategy is to generate root-mean-square magnitude of the stator current $I_{s}^{*}$, and slip frequency $\omega_{s}^{*}$, with the commanded torque and rotor resistance estimate $\hat{r}_{r}$ given. Note that all the stator current can be assumed to be in $q$-axis in the synchronous reference frame through proper choice of phase reference so that $I_{d}^{e^{*}}$ is zero. Thus the relationship of phasor stator current in $q$ - axis and $q / d$-axis current in the synchronous reference frame may be expressed in (5),

$$
\sqrt{2} \tilde{i}_{q s}=i_{q s}^{e}-j i_{d s}^{e}=i_{q s}^{e}
$$

Since the test induction motor is delta-connected, the inverter current command in the synchronous reference frame $i_{q i}^{e^{*}}$ is obtained by scaling the stator current command by $\sqrt{6}$.

The objective of Adaptive MTPA control is to generate torque as close to the torque command as possible and simultaneously to satisfy that generated torque should be maximum for a given stator current. The former is called tracking property and the latter is called maximum torque per amp condition (MTPA condition). These can be formulated as

$$
\left\|T_{e}\left(\omega_{s}^{*}, I_{s}^{*}, \hat{r}_{r}\right)-T_{e}^{*}\right\|<\varepsilon
$$

Herein, $\varepsilon$ is a very small number and

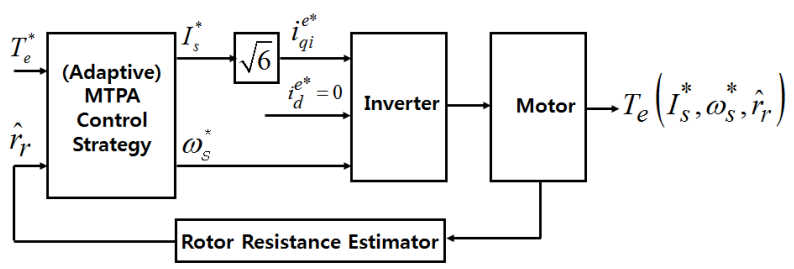

Fig. 3. Structure of adaptive maximum torque per amp control 


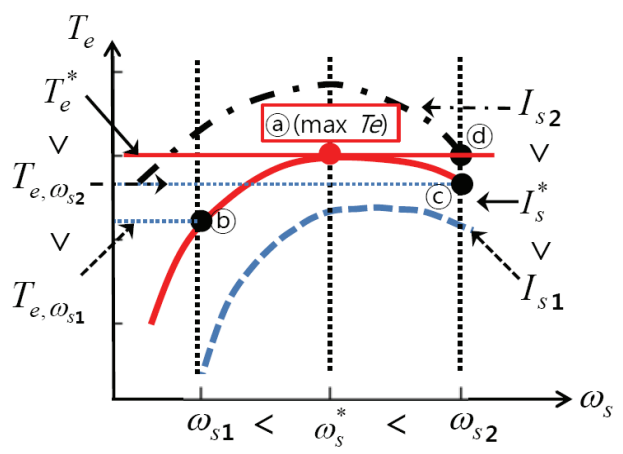

Fig. 4. Objectives of adaptive MTPA control

$$
T_{e}\left(I_{s}^{*}, \omega_{s}^{*}, \hat{r}_{r}\right)=\max _{I_{s}, \omega_{s}}\left\{T_{e}\left(I_{s}, \omega_{s}, \hat{r}_{r}\right)\right\}
$$

In other words, the commanded torque is achieved with minimum stator current, thus minimizing conduction loss. For example, as can be seen in Fig. 4, torques at point (a) is larger than torques at (b) and (c) for the stator current of $I_{s}^{*}$. This means that the objective of Adaptive MTPA control achieves its objective at point (a). Note that even though the desired torque was achieved at point (d) for the stator current of $I_{s 2}$ which is greater than $I_{s}^{*}$, conduction loss is higher.

\subsection{Derivation of torque equation}

The derivation of Adaptive MTPA control strategy is to find the relationships which express rms magnitude of the applied stator current $I_{s}$ and slip frequency $\omega_{s}$ as function of command torque and rotor resistance estimate $\hat{r}_{r}$. To do this, the general electromagnetic torque Eq. (8) in the synchronous reference frame is rewritten in terms of rms magnitude of the applied stator current $I_{s}$, slip frequency $\omega_{s}$, and rotor resistance estimate $\hat{r}_{r}$.

$$
T_{e}=\frac{3}{2} \frac{P}{2}\left(\lambda_{d m}^{e} i_{q s}^{e}-\lambda_{q m}^{e} i_{d s}^{e}\right)
$$

Likewise, relationships between $q$-axis magnetizing flux linkage phasor and the $q$ - and $d$ - axis magnetizing flux linkage in the synchronous reference frame [17] is

$$
\sqrt{2} \tilde{\lambda}_{q m}=\lambda_{q m}^{e}-j \lambda_{d m}^{e}
$$

After the algebraic manipulation of (5), (8), and (9), the electromagnetic torque may be reduced to multiplication of stator current and complex conjugate of magnetizing flux linkage phasor,

$$
T_{e}=\frac{3}{2} P \operatorname{Imag}\left(\overline{\tilde{\lambda}_{q m}} I_{s}\right)
$$

where the overbar ' - ' is symbol of complex conjugate. $\tilde{\lambda}_{q m}$ in equation (10) can be rewritten in terms of rms magnitude of the applied stator current $I_{s}$, slip frequency $\omega_{s}$, and rotor resistance estimate $\hat{r}_{r}$. To do this, Using voltage equation at magnetizing branch From the AQDM steady-state equivalent circuit in Fig. 1, $\tilde{\lambda}_{q m}$ may be expressed as

$$
\tilde{\lambda}_{q m}\left(\omega_{s}, I_{s}, r_{r}\right)=\frac{Z_{a g}\left(\lambda_{m}, \omega_{s}, r_{r}\right)}{j \omega_{e}} I_{s}
$$

where $Z_{a g}$ is the impedance in parallel with two branches, $j \omega_{e} / \Gamma_{m}\left(\lambda_{m}\right)$ and $j \omega_{e} L_{l r}\left(\lambda_{m}\right)+Z_{r}\left(j \omega_{s}\right) / S$, resulting in

$$
Z_{a g}\left(\lambda_{m}, \omega_{s}, r_{r}\right)=\frac{\omega_{e}}{-j \Gamma_{m}\left(\lambda_{m}\right)+\frac{\omega_{s}}{j \omega_{s} L_{l r}\left(\lambda_{m}\right)+Z_{r}\left(j \omega_{s}\right)}}
$$

Thus, the electromagnetic torque can be achieved in terms of $\omega_{s}, I_{s}$, and $r_{r}$ with (11) substituted into $\tilde{\lambda}_{q m}$ in (10), which is

$$
T_{e}\left(\omega_{s}, I_{s}, r_{r}\right)=\frac{3}{2} P \operatorname{Imag}\left(\overline{\left(\frac{Z_{a g}\left(\lambda_{m}, \omega_{s}, r_{r}\right)}{j \omega_{e}} I_{s}\right)} I_{s}\right)
$$

where $\lambda_{m}\left(\omega_{s}, I_{s}, r_{r}\right)$, which is $\sqrt{2}\left|\tilde{\lambda}_{q m}\right|$ can be achieved by solving the voltage equation at magnetizing branch by any nonlinear algebraic equation solver

$$
\left|\omega_{e} \lambda_{m}\right|=\sqrt{2}\left|I_{s} \cdot Z_{a g}\left(\lambda_{m}, \omega_{s}, r_{r}\right)\right|
$$

Herein, Newton-Raphson method was employed to solve $\lambda_{m}\left(\omega_{s}, I_{s}, r_{r}\right)$ in (14).

To find optimal slip frequency, $\omega_{s}^{*}$ for the given stator current, $I_{s}^{*}$, and rotor resistance estimate, $\hat{r}_{r}$, to maximize torque, (13) may be treated to optimization problem in (15). Therein, one data set of maximized torque $T_{e, \text { max }}\left(I_{s}^{*}, \omega_{s}^{*}, \hat{r}_{r}\right)$, optimal slip frequency, $\omega_{s}^{*}$, stator current, $I_{s}^{*}$, and rotor resistance estimate, $\hat{r}_{r}$ can be obtained by any optimization technique.

$$
T_{e, \max }=\underbrace{\max }_{\omega_{s}} \frac{3}{2} P \operatorname{Imag}\left(\overline{\left(\frac{Z_{a g}\left(\lambda_{m}, \omega_{s}, r_{r}\right)}{j \omega_{e}} I_{s}\right)} I_{s}\right)
$$

The acquisition of data set of maximized torque $T_{e, \max }$, optimal slip frequency, $\omega_{s}^{*}$, stator current, $I_{s}^{*}$, and rotor resistance estimate, $\hat{r}_{r}$ will be repeated for all combinations of $I_{s}$ and $r_{r}$. The $j$-th point of $I_{s}$ in the range of from nearly $0 \mathrm{~A}$ to rated current will be denoted $I_{s, j}^{*}$ and k-th point of $r_{r}$ between $0.01 \Omega$ and $0.21 \Omega$ will be denoted $r_{r, k}$ for convenient purposes.

The maximized torque $T_{e, \max , j, k}$ and optimal slip frequency, $\omega_{s, j, k}^{*}$, for a given pair of $\left(I_{s, j}^{*}, r_{r, k}\right)$ can be 


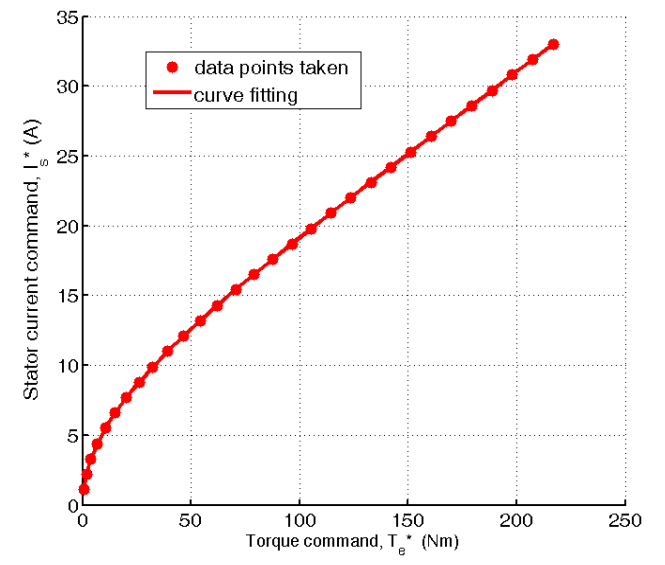

(a) Stator current command versus torque command

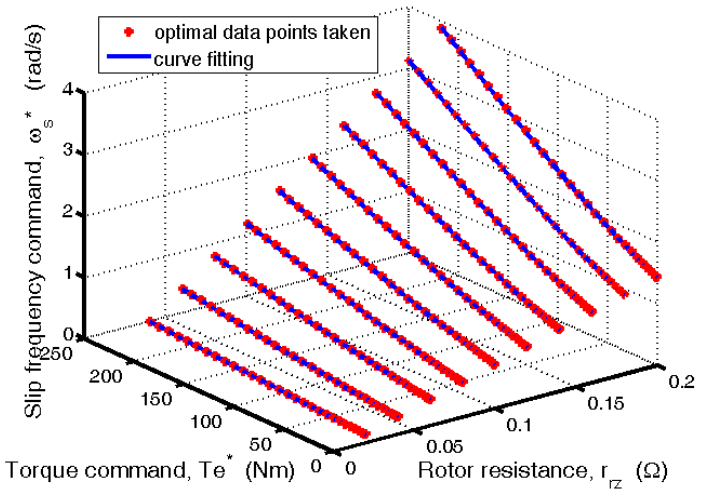

(b) Slip frequency command vs. torque command and rotor resistance

Fig. 5. The control law and data points for Adaptive MTPA control based on AQDM

achieved by solving (15) with $I_{s}$ and $r_{r}$ replaced by $I_{s, j}^{*}$ and $r_{r, k} . \omega_{s, j, k}^{*}$ and $T_{e, \max , j, k}^{*}$ are the resulting optimum slip frequency and the corresponding maximum torque for $I_{s, j}^{*}$ and $r_{r, k}$, respectively. These resulting data points are recorded to find two relationships in Adaptive MTPA control and are plotted in Fig. 5. One interesting observation in Fig. 5 is that $I_{s}$ is not a function of $r_{r}$.

To find the relationship of stator current in terms of torque command, the data points $\left\{I_{s, j}^{*}, T_{e, j, k}^{*}\right\}$ in Fig. 5 (a) are used and curve fitted to the form of the relationship formulated as

$$
I_{s}^{*}\left(T_{e}^{*}\right)=a_{1} T_{e}^{*}+a_{2} T_{e}^{* b_{1}}+a_{3} T_{e}^{* b_{2}}
$$

where $a_{1}, a_{2}, a_{3}, b_{1}$, and $b_{2}$ are parameters to be identified.

As for the relationship of slip frequency $\omega_{s}$ which is highly rotor resistance dependent, the data points $\left\{\omega_{s, j, k}^{*}, T_{e, j, k}^{*}, r_{r, k}\right\}$ are fit to the functional form

$$
\omega_{s, A M T P A}^{*}\left(T_{e}^{*}, r_{r}^{*}\right)=d_{0} r_{r}^{* n 1}+d_{1} r_{r}^{* n 2} T_{e}^{* n 3}
$$

where $d_{0}, d_{1}, n_{1}, n_{2}$, and $n_{3}$ are parameters to be identified.
To obtain coefficients in (16) and (17), any fitting technique could be used. In this work, a genetic algorithm is employed, which was part of the Genetic Optimization System Engineering Tool (GOSET 1.02), a Matlab based toolbox. Details are set forth in [18].

The resulting relationships for $I_{s}^{*}$ and $\omega_{s}^{*}$ for the test machine may be expressed as

$$
\begin{gathered}
I_{s}^{*}\left(T_{e}^{*}\right)=0.102 T_{e}^{*}-6.410 T_{e}^{* 0.011}+7.790 T_{e}^{* 0.152} \\
\omega_{s, \text { AMTPA }}^{*}\left(T_{e}^{*}, r_{r}^{*}\right)=7.22 \cdot r_{r}^{*}+0.025 r_{r}^{*} \cdot T_{e}^{* 1.15}
\end{gathered}
$$

and are also plotted along with the individual data points in Fig. 5. It can be seen that (18) and (19) fit the calculated data points $\left\{I_{s, j}^{*}, \omega_{s, j}^{*}, T_{e, j}^{*}\right\}$ in a good accuracy.

\section{Laboratory Experimental Set-up}

The performance of Adaptive MTPA control strategy at light load was validated through experimental study. In this work, voltage source current controlled inverter was used to drive the induction motor as shown in Fig. 6. To this end, a current controlled inverter-fed drive was used to operate the test induction motor. Therein, Synchronous current regulator (SCR) is used to keep currents into the motor to follow current command and delta modulator to determine the switching signal for IGBTs, $\mathrm{T}_{1} \sim \mathrm{T}_{6}$. Dynamometer is identical to the test induction motor in the chamber and drives the test induction motor. To experience the test induction motor in the light and heavy load, torque reference $T_{e}^{*}$, is commanded by Adaptive or Non-Adaptive

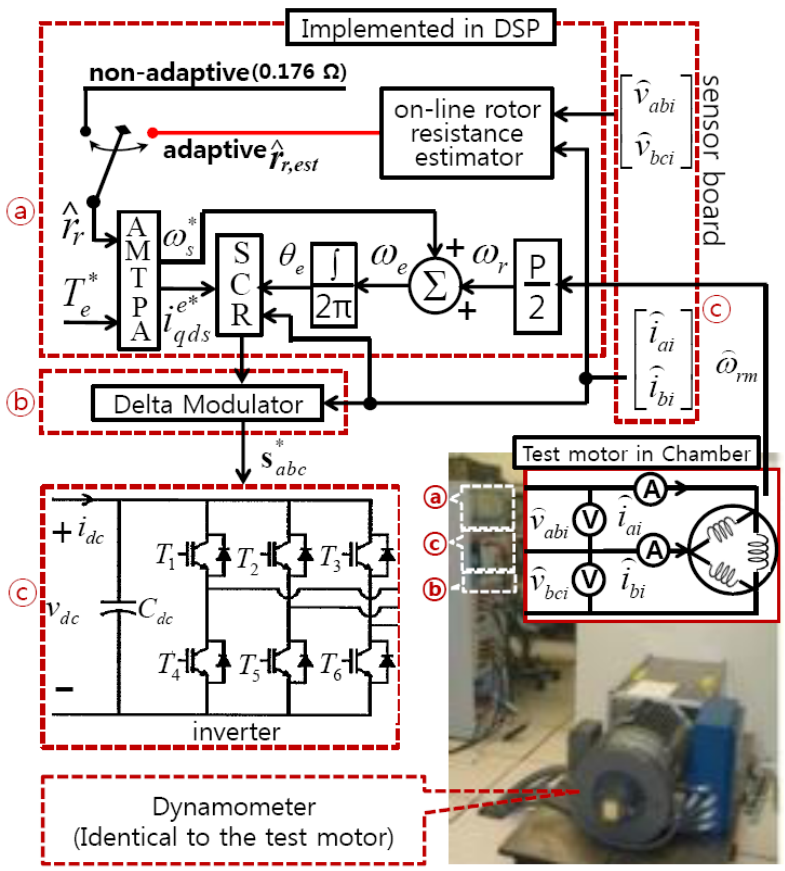

Fig. 6. The experimental set-up for induction machine drive 
MTPA control strategies depending on its load and the speed of the test motor $\widehat{\omega}_{r m}$ is maintained at a speed of $900 \mathrm{rpm}$ by the dynamometer. In this work, $25 \mathrm{Nm}$ is commanded as the torque conference for light load operation which corresponds to about $10 \%$ of $200 \mathrm{Nm}$ of full load torque and $150 \mathrm{Nm}$ for heavy load operation.

As for control strategy, Adaptive MTPA control utilize rotor resistance estimator to estimate the actual rotor resistance because direct measurement of actual rotor resistance is so difficult. Note that the estimated rotor resistance was feedback to compute optimal slip frequency $\omega_{s}^{*}$. Any rotor resistance estimator can be used [19]. To validate the performance of Adaptive MTPA control at light load, performance of Non-Adaptive MTPA control strategy whose slip frequency command $\omega_{s}^{*}$ use the value of $0.176 \Omega$ for rotor resistance in (21) and ends up with

$$
\omega_{s, M T P A}^{*}\left(T_{e}^{*}, 0.176 \Omega\right)=1.2707+0.0044 \cdot T_{e}^{* 1.15}
$$

Note that the value of rotor resistance in $(20)$ is $0.176 \Omega$ when AQDM parameters of the test induction motor was identified at the temperature of $43^{\circ} \mathrm{C}$.

\section{Review of Performance of Adaptive MTPA Control at Heavy Load}

The performance of Adaptive MTPA control at heavy load with torque command of $150 \mathrm{Nm}$ [7] is reviewed in order to give insight on objective of MTPA control strategy.

In Fig. 7, torques generated by the Adaptive MTPA control with the torque command of $150 \mathrm{Nm}$ was redrawn in two temperature regions. One region is the temperature of $43{ }^{\circ} \mathrm{C}$ at which the characterization procedure was carried on for AQDM parameter identification and used in design of Adaptive MTPA control. The other region is room temperature which ranges from $24{ }^{\circ} \mathrm{C}$ to $30{ }^{\circ} \mathrm{C}$. The resultant torque by the Adaptive MTPA control whose slip frequency command is $\omega_{S, A M T P A}^{*}$ in (19) is represented by the red solid line with dotted marks and the resultant one by Non-Adaptive MTPA control whose slip frequency command $\omega_{s, M T P A}^{*}$ in (20) is illustrated by the dark dotted line. Two additional torque measurements by NonAdaptive MTPA control are added at the vicinity of slip frequency command $\omega_{s, M T P A}^{*}$ in (20). Those slip frequencies are 0.9 times and 1.1 times of $\omega_{s, M T P A}^{*}$ in (20). The dashdotted line and dotted line illustrates the torque generated by Non-Adaptive MTPA control at slip frequency of 0.9 times and 1.1 times of $\omega_{S, M T P A}^{*}$ in (20).

In Fig. 7 (a), Non-Adaptive MTPA control generates torque almost equal to torque command of $150 \mathrm{Nm}$ at $43^{\circ} \mathrm{C}$. However, the torque at cold temperature was degraded and more torque and close to torque command is produced at the vicinity of slip frequency command of $\omega_{s, M T P A}^{*}$ which is 0.9 times of $\omega_{s, M T P A}^{*}$ in (20). On the

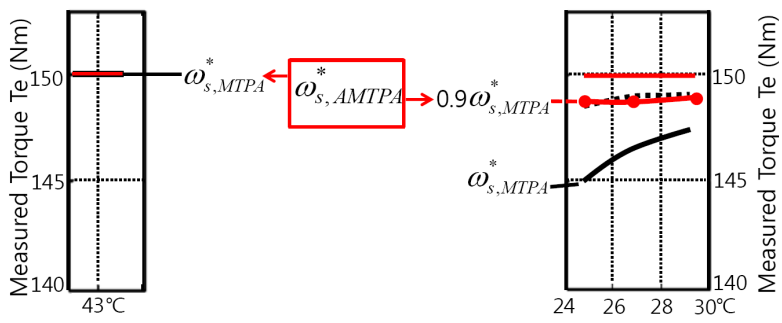

(a) Tracking property

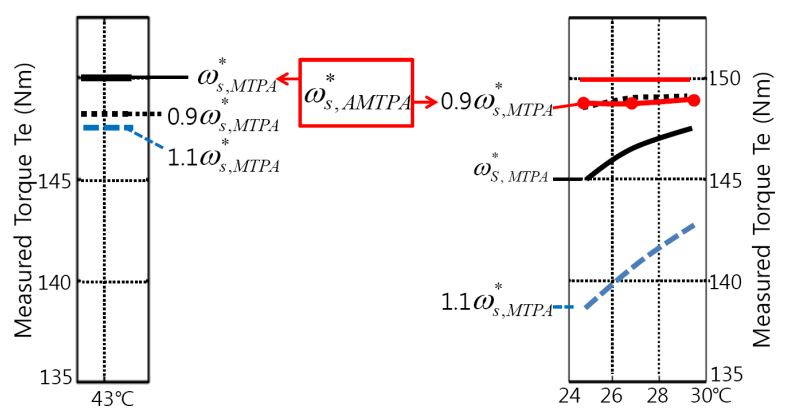

(b) Maximum torque per amp condition

Fig. 7. Performance of Adaptive MTPA control at heavy $\operatorname{load}\left(T_{e}^{*}=150 \mathrm{Nm}\right)$

other hand, Adaptive MTPA control produces torque close to the torque command by varying slip frequency command of $\omega_{s, A M T P A}^{*}$ in (19) by taking rotor resistance variation into account. Note that $\omega_{s, A M T P A}^{*}$ in (19) at $43^{\circ} \mathrm{C}$ is same as $\omega_{s, M T P A}^{*}$ in (20) but it adapted itself to 0.9 times of $\omega_{s, M T P A}^{*}$ in (20) at which more torque is generated.

As for the MTPA condition shown in Fig. 7 (b), Adaptive MTPA control satisfies MTPA condition. The desired torque at $43^{\circ} \mathrm{C}$ is largest at $\omega_{s, A M T P A}^{*}$ in (19) compared to torques at its vicinities. However, while the maximum torque is achieved at $\omega_{s, M T P A}^{*}$ in $(20)$ at $43^{\circ} \mathrm{C}$ by Non-Adaptive MTPA control, the largest torque was generated at 0.9 times of $\omega_{s, M T P A}^{*}$ in (20) at cold temperature. The interesting observation on $\omega_{s, A M T P_{A}}^{*}$ in (19) is that $\omega_{s, A M T P A}^{*}$ in (19) adjusted itself from $\omega_{s, M T P A}^{*}$ in (20) at $43^{\circ} \mathrm{C}$ to 0.9 times of $\omega_{s, M T P A}^{*}$ in (20) at cold temperature by considering rotor resistance variation.

\section{Study on Performance of Adaptive MTPA Control at Light Load}

The performance of Adaptive MTPA control at light load (herein, $25 \mathrm{Nm}$ ) was compared in the same way as did in Section 5. As can be seen in Fig. 8 (a), the torque by Non-Adaptive MTPA control at optimal slip frequency command, $\omega_{s, M T P A}^{*}$, in (20) at light load is generated by $4 \%$ error from torque command even at temperature of $43^{\circ} \mathrm{C}$. This error may be tolerable with the operation at light load where lower signal to noise ratio, small offset and error in sensors have poorer influence on performance than at heavy load. This is same case for Adaptive MTPA control 


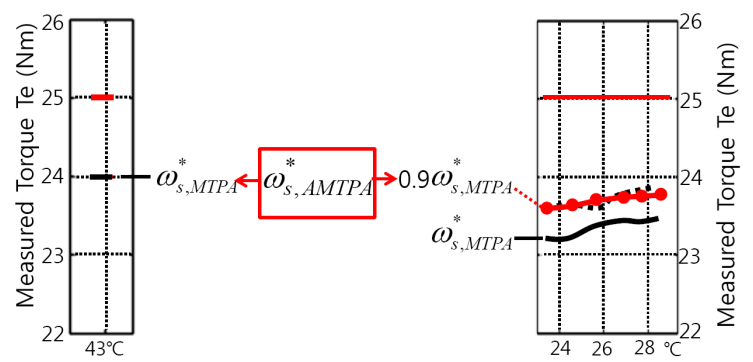

(a) Tracking property

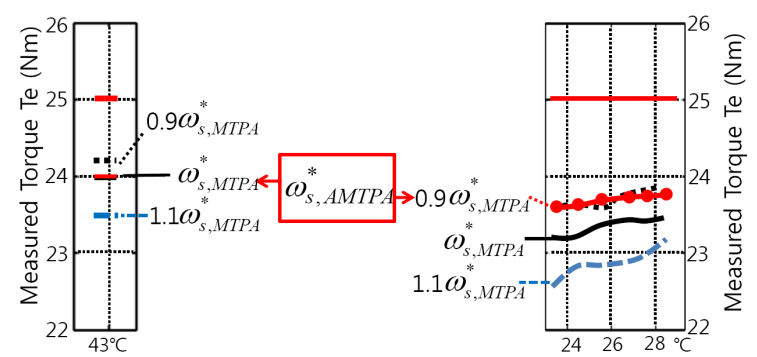

(b) Maximum torque per amp condition

Fig. 8. Performance of Adaptive MTPA control with $T_{e}^{*}=$ $25(\mathrm{Nm})$ at cold temperature

since optimal slip frequency command, $\omega_{s, A M T P A}^{*}$, in (19) is identical to $\omega_{s, M T P_{A}}^{*}$, in (20) since the rotor resistance estimate used in $\omega_{s, A M T P A}^{*}$, in (19) is same as $0.176 \Omega$.

However, superior performance of Adaptive MTPA control over the performance of Non-Adaptive MTPA control can be seen in measurement at cold temperature as well. In Fig. 8 (a), the torque by Non-Adaptive MTPA control is degraded but the torque by Adaptive MTPA control is still tracking the torque produced by NonAdaptive MTPA control at temperature of $43^{\circ} \mathrm{C}$ with little degradation. Therein, optimal slip frequency, $\omega_{s, A M T P A}^{*}$ of Adaptive MTPA control given by (19) is found equal to 0.9 times of $\omega_{s, M T P A}^{*}$ at which the largest torque was generated by Non-adaptive MTPA control. This means that Adaptive MTPA control strategy adjusted the slip frequency command, $\omega_{s, A M T P A}^{*}$, in (19) so that the largest torque are always achieved at $\omega_{s, A M T P A}^{*}$ by taking rotor resistance variation into an account.

As for the MTPA condition in Fig. 8 (b), because of inherent error at light load, the torque generated at $\omega_{s, M T P A}^{*}$, in (20) is not largest but still close to the largest torque at 0.9 times of $\omega_{s, M T P A}^{*}$, in (20). This claim can be arguably. However, satisfaction of MTPA condition can be seen obvious at cold temperature. Optimal slip frequency of $\omega_{s, A M T P A}^{*}$ in (19) adjusted itself to 0.9 times of $\omega_{s, M T P A}^{*}$, in (20) to produce larger torque at three resultant torque. This observation justifies that Adaptive MTPA control still works at light load so that its two objectives, tracking property and MTPA condition, are achieved.

The observations from Fig. 8 (b) indicate that the Adaptive MTPA control strategy seeks to achieve a desired torque with the minimum possible stator current at light load as well as at heavy load regardless of rotor temperature variation.

\section{Conclusion}

It was experimentally shown that the Adaptive MTPA control strategy performs optimally at light load regardless of rotor resistance variation by showing that the optimal slip frequency seeks for optimal slip frequency at which the torque produced is always the largest and desirable at the same time.

As can be seen from experimental results in the previous section, the Adaptive MTPA control strategy makes true optimal performance at light loads regardless of rotor resistance variation by reflecting rotor resistance variation in the design of optimal slip frequency control law.

It can be conclude that performance of Adaptive MTPA control strategy is still consistent at light loads as well as at heavy loads

\section{Acknowledgements}

This research was supported in part by Basic Science Research Program through the National Research Foundation of Korea(NRF) funded by the Ministry of Education, Science and Technology (Grant 20130235 ) and was supported in part by the Soonchunhyang University Research Fund

\section{References}

[1] Y. Zhang, "Induction Motor with Adjustable Windings for High Efficiency Drive in Light Load Operation," Journal of Electrical Engineering, Vol. 9, No.2, 2014, pp. 508-513.

[2] M. Farasat, A. Trzynadlowski, M. Fadali, "Efficiency improved sensorless control scheme for electric vehicle Induction Motors," IET Electrical Systems in Transportaion, Dec 2014, pp. 122-131.

[3] F. Ferreira, J. Simoes, J. Oliverira, "Novel Electronic Device to Improve the Performance of VariableTorque Fixed-Speed Induction Motors," $20159^{\text {th }}$ International Conference on Compatibility and Power Electronics (CPE), 2015, pp. 281-288.

[4] A. Odhano, R. Bojoi, A. Boglietti, S. Rosu, G. Griva, "Maximum Efficiency per Torque Direct Flux Vector Control of Induction Motor Drives," IEEE transactions on Industry Applications, Vol. 51, No. 6, 2015, pp. 4415-4424.

[5] Y. Liu and A. Bazzi, "A comprehensive Analytical Power Loss Model of an Induction Motor Drive System with Loss Minimization Control," 2015 IEEE International Electric Machines \& Drives Conference 
(IEMDC), May 2015, pp. 1638-1643

[6] X. Fu and S. Li, "A Novel Neural Network Vector Control Technique for Induction Motor Drive," IEEE Transactions on Energy Conversion, Vol. 30, No. 4, Dec 2015, pp.1428- 1437

[7] C. Kwon, "Study on Optimal Condition of Adaptive Maximum Torque Per Amp Controlled Induction Motor", Journal of Electrical Engineering Technology, Vol. 9, No. 1, January, 2014, pp. 231-238.

[8] C. Kwon, "Study on an Adaptive Maximum Torque Per Amp Control Strategy for Induction Motor Drives," Journal of Electrical Engineering \& Technology, Vol. 8, No. 1, 2013, pp. 110-117.

[9] O. Wasynczuk, S. D. Sudhoff, K. A. Corzine, J. L. Tichenor, P.C. Krause, I.G. Hansen, and L.M. Taylor, "A Maximum Torque Per Ampere Control Strategy for Induction Motor Drives," IEEE Transactions on Energy Conversion, Vol. 13, No. 2, June 1998, pp. 163-169.

[10] S. Krim, S. Gdaim, A. Mtibaa, M. Mimouni, "Design and Implementation of Direct Torque Control Based on an Intelligent Technique of Induction Motor on FPGA," Journal of Electrical Engineering, Vol. 10, No. 4, 2015, pp. 1527-1539.

[11] I. Jeong, W. Choi, K. Park, "Sensorless Vector Control of Induction Motors for Wind Energy Applications Using MRAS and ASO", Journal of Electrical Engineering Technology, Vol. 9, No. 3, 2014, pp. 873-881.

[12] M. Comanescu, “A Robust Sensorless Sliding Mode Observer with Speed Estimate for the Flux Magnitude of the Induction Motor Drive," $20159^{\text {th }}$ International conference on Compatibility and Power Electronics (CPE), 2015, pp. 224-229.

[13] S. Jurkovic, K. Rahman, J. Morgante, P. Savagian, "Induction Machine Design and Analysis for General Motors e-Assist Electrification," IEEE Transaction on Industry Applications, Vol. 51, No. 1, Jan/Feb 2015, pp. 631-639.

[14] S. D. Sudhoff, P. L. Chapman, D. C. Aliprantis, and B. T. Kuhn, "Experimental Characterization Procedure for Use with of an Advanced Induction Machine Model," IEEE Transactions on Energy Conversion, Vol. 18, Mar 2003, pp. 48-56.

[15] S. D. Sudhoff, D. C. Aliprantis, B. T. Kuhn, and P. L. Chapman, "An Induction Machine Model for Predicting Inverter - Machine Interaction," IEEE Transactions on Energy Conversion, Vol. 17, June 2002, pp. 203-210

[16] C. Kwon, S. D. Sudhoff, "Genetic Algorithm-Based Induction Machine Characterization Procedure With Application to Maximum Torque Per Amp Control," IEEE Transactions on Energy Conversion, Vol. 21, June 2006, pp. 405-415

[17] P. C. Krause, O. Wasynczuk, S. D. Sudhoff, Analysis of Electric Machinery and Drive Systems, IEEE
Press, 2002.

[18] "Energy Systems Analysis Consortium (ESAC) Genetic Optimization System Engineering Tool (GOSET) Ver 1.02," School of Electrical and Computer Engr., Purdue Univ., West Lafayette, IN, 47907, 2003.

[19] C. Kwon, S. D. Sudhoff, “An On-line Rotor Resistance Estimator for Induction Machine Drives," the 2005 International Electric Machines and Drives Conference, May 2005, pp. 391-397.

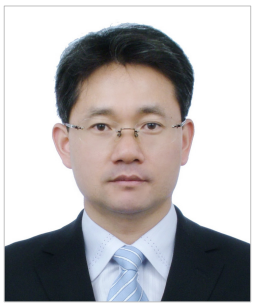

Chun-Ki Kwon received B.S. and M.S. degrees in electrical engineering from Korea University in 1992 and 1994, respectively. He is with Soonchunhyang University, Asan, Chungnam, Korea. His research interests include control and modeling of electric machines, and expands this area to medical engineering such as rehabilitation devices.

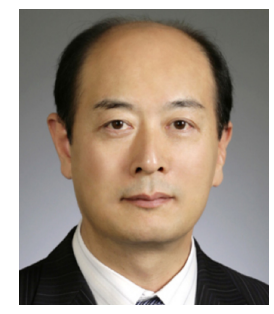

Yong-Hae Kong received B.S. degree in electronic Engineering from Yeonsei University in 1982, and M.S. and Ph.D degrees in Computer Science from New York University (Polytechnic Institute) in 1986, and 1991, respectively. He is with Soon-chunhynag University, Asan, Chungnam, Korea since 1991.

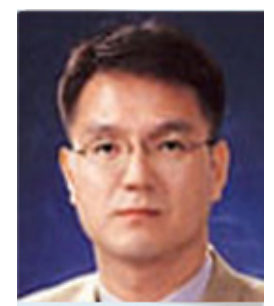

Dong-Sik Kim received B.S., M.S., $\mathrm{Ph} . \mathrm{D}$ degrees in electrical engineering from Korea University in 1986, 1988, and 1992, respectively. He is professor in the department of Electrical Engineering, Soonchunhyang University, Asan, Chungnam, Korea, since 1992. His research interests are web-based virtual laboratory, distance education, nonlinear robust control and intelligence control. 